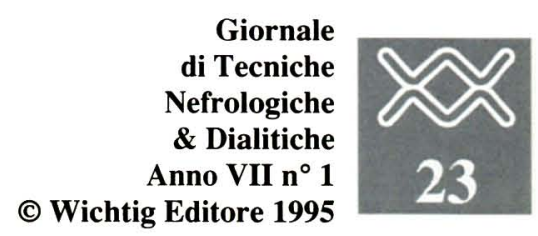

Giornale
di Tecniche
Nefrologiche
\& Dialitiche
nno VII $n^{\circ} 1$
Editore 1995

\title{
Le nuove frontiere dell'imaging diagnostico nella valutazione anatomica del rene: confronto tra US, Angio-TC e RM
}

\author{
A. Valli' ${ }^{1}$ P. Peterlongo ${ }^{2}$, S.W. Della Sala ${ }^{2}$, M. Niccolini ${ }^{2}$ \\ ${ }^{1}$ Divisione di Nefrologia e Dialisi \\ ${ }^{2}$ Unità Operativa di Radiodiagnostica, Ospedale Santa Chiara, USL C5 - Trento
}

copo di questo lavoro è definire il ruolo delle singole metodiche di imaging diagnostico nello studio dell'anatomia renale.

Molte sono le vie attraverso le quali si può arrivare ad ottenere un imaging anatomico, ma le informazioni ottenute possono essere diverse a seconda della metodica utilizzata: esse possono quindi risultare difformi da quanto si vuole conoscere se non si finalizza l'indagine allo scopo di ottenere l'informazione più utile alla soluzione del quesito diagnostico. Sono ormai note a tutti le metodiche di imaging più diffuse, ovvero gli ultrasuoni, la TAC e la RM. La metodica più familiare è l'ecotomografia. Essa, come è noto, presenta dei grossi vantaggi. È una metodica del tutto innocua, con un basso costo, determinato sia dal valore relativamente contenuto delle apparecchiature, sia dalla possibilità di sottoporre all'indagine un numero di pazienti molto elevato. L'apparecchiatura per ecotomografia è attualmente capillarmente diffusa ed è spesso ubicata, oltre che presso strutture prettamente deputate alla diagnostica per immagini, anche presso reparti di degenza. L'innocuità dell'esame consente altresì una sua facile ripetibilità nel caso che sorgano dei dubbi di valutazione.

Ma la metodica ecotomografica offre anche dei vantaggi nell'ambito dell'imaging strettamente detto, ovvero una elevata affidabilità nella valutazione della morfologia e delle dimensioni del rene. Inoltre è nota la sua elevata sensibilità verso lesioni focali.

Ovviamente l'ecotomografia presenta degli svantaggi, essendo una metodica non particolarmente sofisticata. Essa non consente per esempio una raffinata valutazione delle strutture vascolari venose e arteriose del rene, quindi del peduncolo vascolare renale nel suo complesso. Inoltre, come noto, l'ecotomografia pecca di panoramicità, offre informazioni del tutto settoriali e quindi spesso non consente di stabilire gli esatti rapporti tra una lesione renale e le strutture adiacenti al rene.

Altra metodica a disposizione del diagnosta è la TAC convenzionale. Perché si parla di TAC convenzionale? Tale termine è entrato in uso recentemente, dopo che la TAC ha subito una notevole evoluzione tecnica nella cosiddetta TAC volumetrica, della quale si parlerà successivamente. Il termine TAC convenzionale si riferisce alla massima parte delle apparecchiature per TAC finora disponibili nelle varie strutture ospedaliere.

La TAC offre ovviamente una serie di vantaggi. Data la diffusione delle apparecchiature, il costo per esame è abbastanza contenuto ed è possibile realizzare tale indagine con una certa facilità, senza significative attese.

Dal punto di vista dell'imaging strettamente detto, la TAC consente una ottima visualizzazione del parenchima renale ed un buon riconoscimento dei piani di clivaggio rispetto alle strutture adiacenti, soprattutto se si usa mezzo di contrasto iodato. In particolare, è possibile definire soprattutto sul piano assiale le dimensioni renali. Inoltre l'uso, ormai ben definito nella quantità e nel modo di somministrazione, del mezzo di contrasto endovena consente discrete informazioni funzionali sul rene.

La TAC offre inoltre un corredo di informazioni più che soddisfacente sui rapporti tra l'eventuale lesione renale e tutte le strutture parenchimatose e vascolari adiacenti. Notevole è la sua sensibilità verso lesioni focali del parenchima renale.

Ovviamente la TAC è metodica radiologica e quindi usa radiazioni ionizzanti. Questo è uno svantaggio. La stessa necessità di utilizzazione di mezzo di contrasto somministrato per via endovena comporta un certo grado di rischio. Questi due fattori negativi non rendono l'esame ripetibile in tempi brevi.

Un ulteriore svantaggio di questa metodica sta nell'impossibilità di ottenere delle informazioni corrette sulle dimensioni del rene secondo il diametro polopolare. Infatti non è possibile ottenere delle immagini dirette del rene secondo questo asse e le ricostruzioni elettroniche, che tutte le macchine consentono, non offrono nella quasi totalità dei casi delle informazioni corrette sulle dimensioni renali. Inoltre scarsamente valuta- 




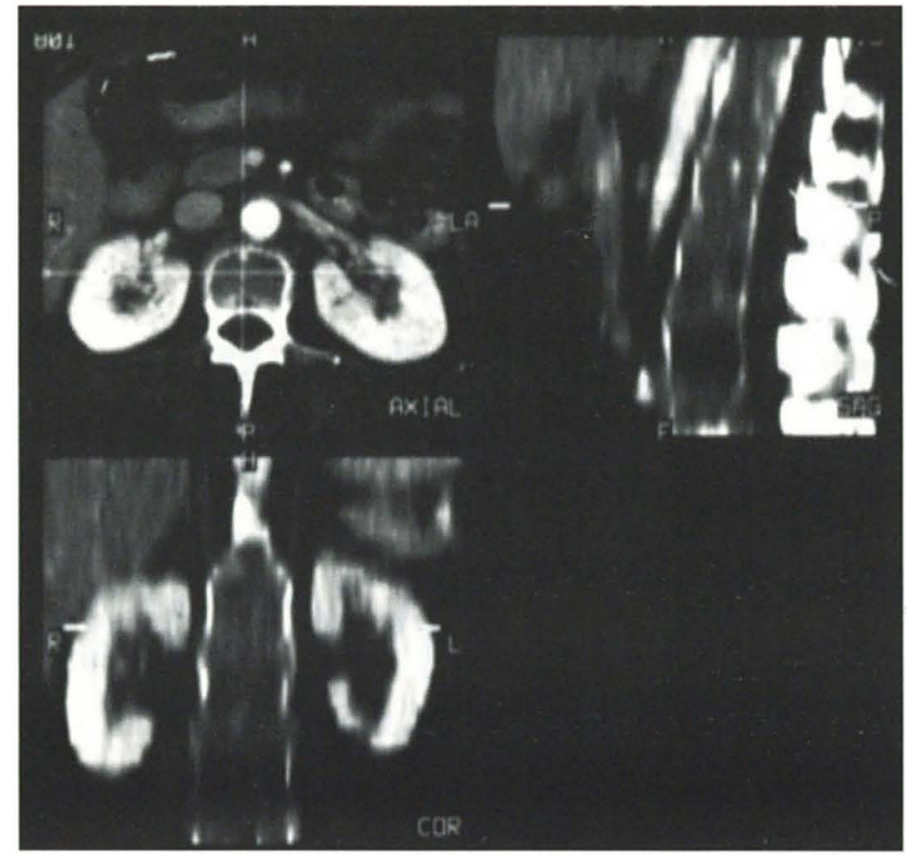

Fig. 1 - Immagine eseguita con TAC volumetrica: si noti come in tempo reale si possano ottenere immagini assiali, coronali o sagittali.

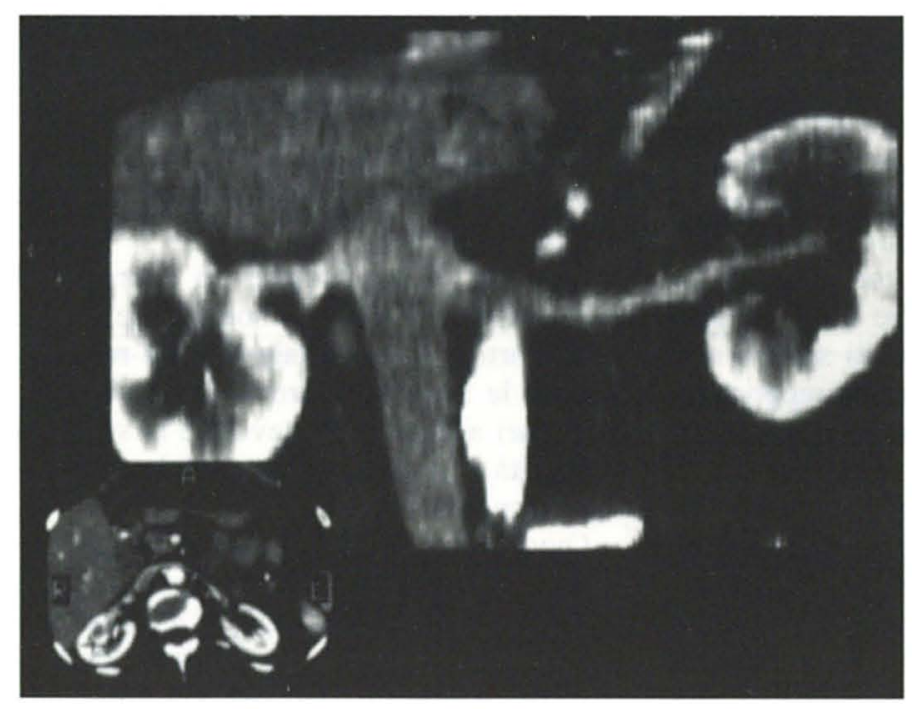

Fig. 3 - Le immagini renali mediante RM: immagine di un rene in coronale con una tecnica che serve per la valutazione morfologica ed all' evidenziazione di piccole lesioni focali.

bile con la TAC convenzionale è la situazione delle strutture vascolari renali e quindi del peduncolo vascolare.

Una autentica rivoluzione nell'ambito della TAC è data dal fatto che da pochi mesi sono state messe a disposizione delle macchine che consentono la cosiddetta acquisizione volumetrica, ovvero acquisiscono i dati riguardanti la regione

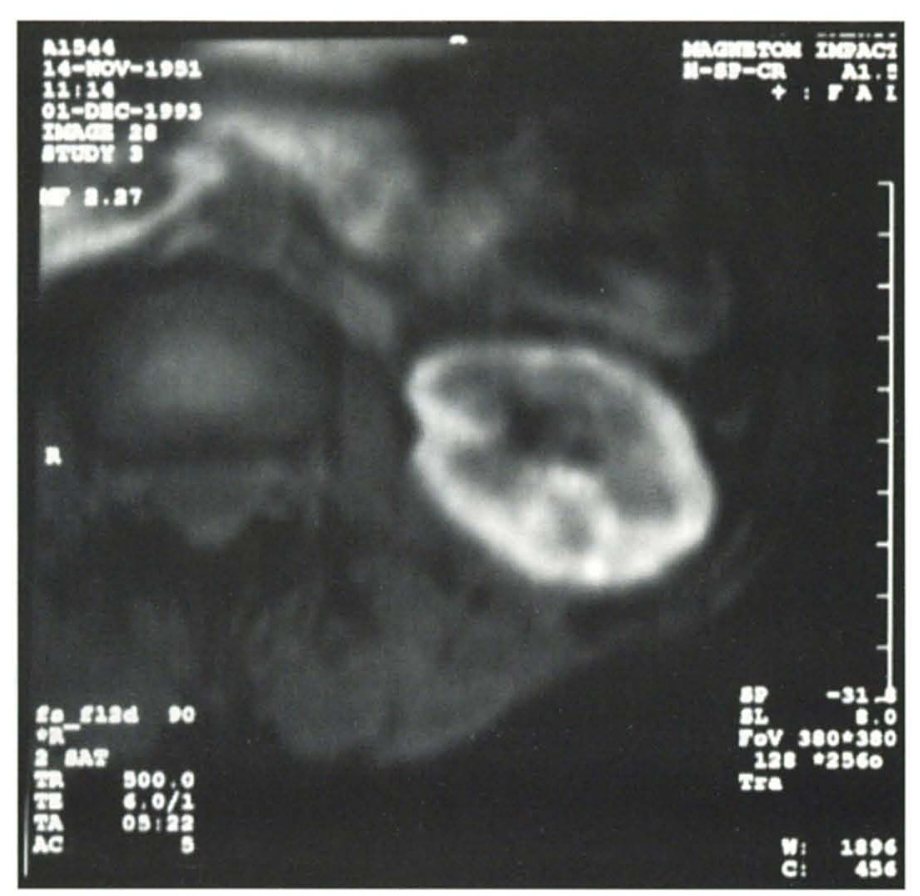

Fig. 2 - Altro grosso vantaggio con la TAC volumetrica con ricostruzione multiplanare, sono le informazioni ottenute sul peduncolo vascolare; in questo caso la ricostruzione delle vene renali.

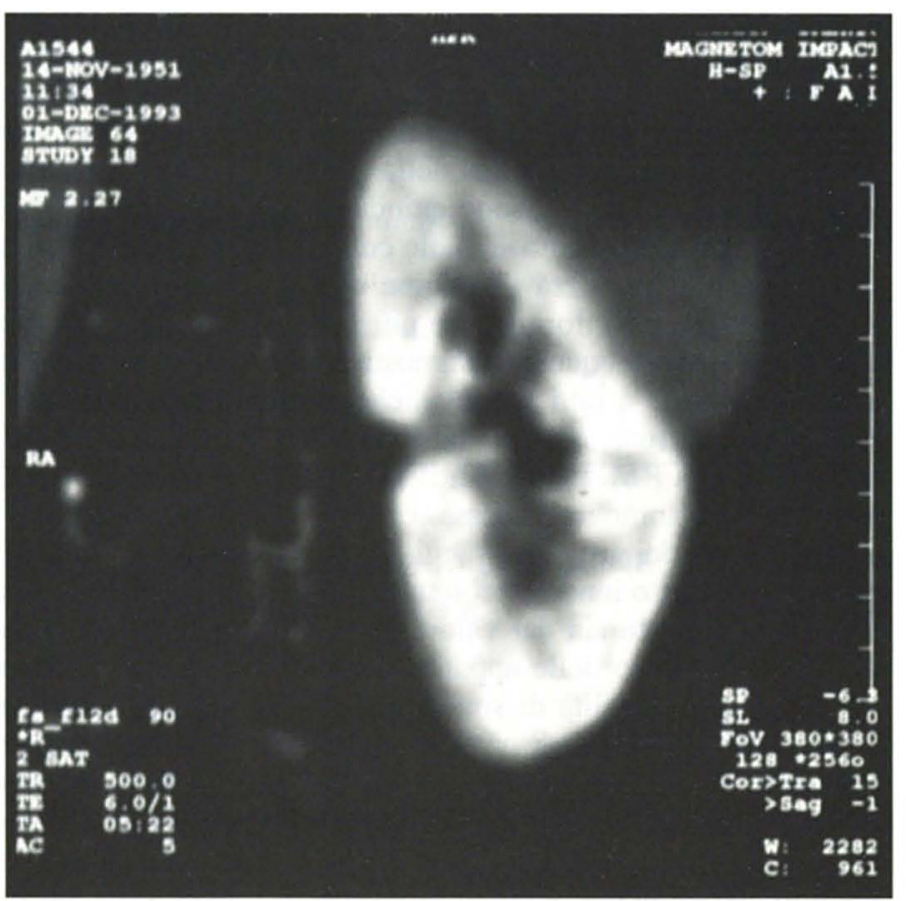

Fig. 4 - Immagine RM del rene eseguita a livello dell' ilo secondo un piano assiale: si possono notare anche fini particolari parenchimali.

studiata sotto forma di un pacchetto a forma di parallelepipedo, entro il quale il calcolatore individua i dati necessari a ricostruire l'immagine di una sezione dell'oggetto studiato secondo qualsivoglia piano dello spazio (Fig. 1).

E intuitivo che tale metodica consente complete informazioni sulle dimensioni renali. Inoltre l'acquisizione a pacchetto dei dati avviene in tempi assai rapidi, 
dell'ordine delle poche decine di secondi. Ciò permette di ottenere notizie di tipo funzionale su tutto il parenchima renale, in quanto è possibile raccogliere informazioni nella fase arteriosa, nella fase corticale, nella fase parenchimale e nella successiva fase venosa trattenendo il paziente sull'apparecchiatura per un tempo brevissimo. Tale riduzione dei tempi di acquisizione consente inoltre una riduzione della quantità di mezzo di contrasto e della dose di radiazioni ionizzanti somministrate al paziente.

Altri vantaggi offerti dalla TAC volumetrica con ricostruzione multiplanare sono ottime informazioni sulle strutture del peduncolo vascolare (Fig. 2) ed una facile dimostrabilità dei rapporti tra eventuali lesioni renali e strutture adiacenti.

Ovviamente le macchine per TAC volumetrica sono assai scarsamente diffuse. $\mathrm{Ne}$ esistono poche in Europa. Qualche mese fa l'Ospedale di Trento ne è stato dotato e ciò ci ha consentito le prime esperienze riguardo a questa nuova tecnica.

La RM è metodica introdotta in tempi relativamente recenti. È noto a tutti che tale metodica non utilizza radiazioni ionizzanti ed è quindi del tutto innocua per $\mathrm{i}$ pazienti.

I grossi vantaggi della RM sono la possibilità di ottenere, come peraltro con la TAC volumetrica, immagini dirette del rene secondo qualsiasi piano spaziale (Figg. 3 e 4). Tale possibilità viene definita multiplanarietà. Ma è noto a tutti che la RM presenta inoltre una sensibilità assai elevata, forse la massima fino ad oggi possibile, verso fini lesioni strutturali dei parenchimali, sia focali che diffuse. Tale proprietà è definita multiparametricità. In un futuro prossimo si pensa che la RM consentirà inoltre una ottimale valutazione delle strutture vascolari renali, del tutto simile a quella della TAC volumetrica e non significativamente diversa da quella consentita dall'angiografia.

Ovviamente la RM è apparecchiatura con un elevatissimo costo di acquisto e quindi non è diffusa capillarmente. L'esame è estremamente costoso, ha una lunga durata, non è del tutto agevole per il paziente e quindi ne è sconsigliabile l'utilizzazione nei casi in cui sia possibile risolvere in maniera adeguata il problema diagnostico con l'ecografia e con la TAC. Inoltre l'indagine con RM richiede la massima collaborazione del pa- ziente perché anche piccoli movimenti semplicemente legati alla respirazione possono compromettere una corretta valutazione del quadro.

Per riassumere quanto detto si può ricordare che l'ecotomografia offre soprattutto la possibilità di una rapida, agevole, semplice valutazione morfo-dimensionale del rene.

La TAC volumetrica consente un passo in avanti, ovvero una valutazione anatomica, anzi, direi, anatomo-funzionale globale del rene, in quanto offre informazioni sulla funzionalità del parenchima renale congiunte ad informazioni sulle strutture vascolari del peduncolo renale.

La RM infine va riservata al momento in cui siano indispensabili fini valutazioni su alterazioni strutturali diffuse o focali del parenchima renale.

L'iter diagnostico che noi ci sentiamo di proporre è pertanto quello che prevede in primo approccio l'ecografia, in seconda istanza, ma con possibilità di informazione assai complete, la TAC; alla RM va riservato un ruolo di complemento alle due prime indagini, qualora siano necessarie fini valutazioni sulla struttura parenchimale. 


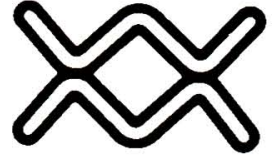

NEWS

a cura di

Umberto Buoncristiani

e Nicola Di Paolo

copertina in cartoncino

plastificato pp. 422

L. 80.000

\section{Wichtig Editore}

Via Friuli, 72/74

20135 MILANO

Tel. 02/55195443

15455122

Fax 02/55195971

INDICE

Informatica e teledialisi

Impiego della cartella clinica computerizzata nella gestione del paziente in dialisi

F.Quarello, G.B. Piccoli, M. Borca, et al

Il computer: principi e applicazioni

G. Quintaliani, A. Orecchini, U. Buoncristiani

La raccolta dei dati nel corso della seduta di dialisi

A. Ramunni, F.P. Schena

L'interesse per un registro policentrico di dialisi e trapianto: l'esempio piemontese

G. Piccoli, M. Salomone, F. Quarello, et al

Uso del registro nel singolo centro

G. Quintaliani, R. Ricci, A. Orecchini, et al

Applicazioni dell'informatica alla dialisi peritoneale

F. Bonello, G.B. Piccoli, F. Quarello, et al

Monitoraggio continuo del sodio in dialisi

S. Di Giulio, L. Meschini, C. Cherubini, et al

Monitoraggio in continuo dei fluidi corporei e del

volume ematico

A. Santoro, E. Mancini

Monitoraggio in continuo delle variazioni

dell'urea mediante bio-sensori per il

controllo dell'adeguatezza dialitica

$S$. Mandolfo, E. Imbasciati

Controllo on-line della temperatura del bagno di

dialisi e stabilità cardio-vascolare

F. Pizzarelli, T. Cerrai, P. Dattolo, et al

Integrazione operativa tra segnali / macchina e

segnali/paziente: proposte applicative

G. Colasanti, G. Arrigo, R. Bucci, et al

Regolazione renale del metabolismo fosfo-calcico

Il controllo renale di calcio e fosforo

G. Mioni, P. Messa, G. Boscutti, et al

\section{TECNICHE}

\section{4}
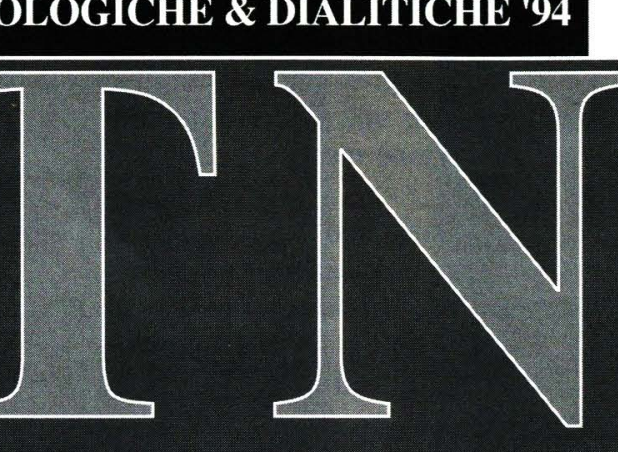

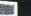

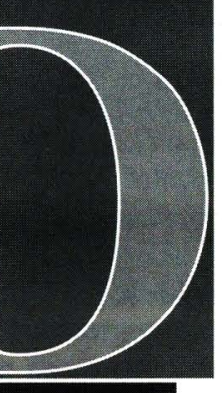

a cura di

Umberto Buoncristiani e Nicola Di Paolo$$
\text { (n) }
$$

Sindromi da alterazione primitiva del controllo renale del metabolismo fosfo-calcico

G. Graziani, S. Finazzi, G. Como

La secrezione del paratormone nell'insufficienza renale cronica e nell'uremia: caratteristiche fisiopatologiche e implicazioni cliniche

D. Rolla, E. Moriero, G. Cannella

Fisiopatologia, prevenzione, terapia medica delle alterazioni del metabolismo fosfo-calcico nella IRC

P. Messa, G. Mioni

Fisiopatologia, prevenzione, terapia medica delle alterazioni del metabolismo fosfo-calcico nell'uremico in dialisi extracorporea

M. Surian, B. Dozio, G. Bonforte, et al

Fisiopatologia, prevenzione, terapia medica delle alterazioni del metabolismo fosfo-calcico

nell'uremico in CAPD

R. Scanziani, B. Dozio, G. Bonforte, et al

Terapia chirurgica ed alcoolizzazione nell'iperparatiroidismo refrattario alla terapia medica

A. Giangrande

Il paratormone come tossina uremica

A. Antonelli, D. Angelini, A. Carlini, et al

Rene ed equilibrio acido-base

Fisiologia della regolazione renale dell'equilibrio acido-base

G.Pontoriero, G. Campolo, F. Locatelli Difetti primitivi della regolazione renale dell'equilibrio acido-base

G.B. Capasso, F. Mollica, C. Saviano

Alterazioni dell'equilibrio acido-base in corso di insufficienza renale acuta

G. Splendiani, G. Barbera, M. Montella
Equilibrio acido-base nell'insufficienza renale cronica (IRC)

D. Cresseri, G. Buccianti, B. Ravasi

Controllo dell'equilibrio acido-base nella dialisi extracorporea

B. Agazia, M. Chellini, O. Garizzo, et al

Le diverse soluzioni tecniche per la bicarbonato-dialisi

Il concentrato di bicarbonato standard

C. Tetta, E. Tessore, C. Bergamini, et al

Bicarbonato solido in cartuccia di polipropilene M. Arati

Bicarbonato sterile in sacca

E. Petrella

Soluzioni per emofiltrazione con sodio bicarbonato in sacca a doppio scomparto in materiale plastico pluristrato "pluricon": relazione tecnica

M. Bottura, R. Remondini, E. Tobaldin

Prescrizione e sorveglianza dell'erogazione del bicarbonato in AFB

M. Atti

Controllo dell'equilibrio acido-base nella CAPD

M. Feriani, G. La Greca

Effetto dei tamponi sulla biocompatibilità delle soluzioni per dialisi peritoneale

N. Di Paolo, G. Garosi, M. Biagioli

Le diverse soluzioni tecniche per l'uso del bicarbonato in CAPD

CAPD con bicarbonato: la soluzione Fresenius M.T. Parisotto

Esperienza clinica con una soluzione di dialisi peritoneale con bicarbonato

S. Rimorini

\section{Il filtro per emodialisi}

Evoluzione della terapia dialitica moderna C. Ronco

Caratteristiche generali delle membrane per emodialisi

R. Tarchini, P. Botti, E. Saccardi, et al

Sistemi di sterilizzazione del filtro

G. Bellinghieri, V. Savica, P. Favazzi

Il lavaggio del filtro: regolamentazione e sistemi

C. Carobi, U. Buoncristiani, R.M. Fagugli

\section{Il liquido per emodialisi}

La qualità dell'acqua nella dialisi contemporanea

E. Lusvarghi

Aspetti tecnici e microbiologici del trattamento dell'acqua di rete per dialisi

G. Cappelli

Filtrazione del liquido di dialisi

per la rimozione di pirogeni

C. Tetta, G. Cappelli, E. Lusvarghi

\section{Dislipidemia e nefropatie}

Fisiopatologia delle alterazioni lipoproteiche nei soggetti affetti da sindrome nefrosica

M.G. Gentile, G. D'Amico

Prevenzione e terapia della dislipidemia

P. Cappelli, L. Di Liberato, F. Sammartino, et al Cause e conseguenze della dislipidemia nell'uremico cronico, nel dializzato e nel trapiantato

R.M. Fagugli, U. Buoncristiani

Costi economici e sociali del trattamento dialitico - Prospettive di contenimento

Analisi dei costi della dialisi extracorporea

R. Ragni, M. Formica, L. Funaro

Costi della dialisi peritoneale

A. Ramello

Il costo sociale della dialisi

G. Ruggieri

Necessità e prospettive di contenimento dei costi della terapia dialitica

U. Buoncristiani 